\title{
Influence of structural and magnetic properties in the heating performance of multicore bioferrofluids
}

\author{
R. Bustamante, ${ }^{*}$ A. Millán, ${ }^{\dagger}$ R. Piñol, and F. Palacio \\ Instituto de Ciencia de Materiales de Aragón, CSIC - Universidad de Zaragoza, Departamento de Física de la Materia Condensada, \\ Facultad de Ciencias, 50009 Zaragoza, Spain \\ J. Carrey and M. Respaud \\ Université de Toulouse; INSA; UPS; LPCNO (Laboratoire de Physique et Chimie des Nano-Objets), 135 Avenue de Rangueil, \\ F-31077 Toulouse, France and CNRS; UMR 5215; LPCNO, F-31077 Toulouse, France \\ R. Fernandez-Pacheco \\ Aragon Institute of Nanoscience (INA), University of Zaragoza, Pedro Cerbuna 12, 50009 Zaragoza, Spain \\ N. J. O. Silva \\ Departamento de Física and CICECO, Aveiro Institute of Nanotechnology, Universidade de Aveiro, 3810-193 Aveiro, Portugal
}

(Received 3 July 2013; revised manuscript received 3 September 2013; published 6 November 2013)

\begin{abstract}
Biomedical applications of superparamagnetic iron oxide particles have been of interest for quite a number of years. Recent developments show that multifunctionality can be efficiently achieved using polymers to coat the particles and to provide anchoring elements to their surface. This leads to the formation of nanobeads with a reduced number of particles trapped by the polymeric structure. While the magnetothermic behavior of isolated nanoparticles has been a subject of interest over the past several years, multicore magnetic nanobeads have thus far not received the same attention. The influence of structural and magnetic properties in the hyperthermia performance of a series of magnetic fluids designed for biomedical purposes is studied here. The fluids are made of maghemite multicore polymeric beads, with variable nanoparticle size and hydrodynamic size, dispersed in a buffer solution. The specific loss power (SLP) was measured from 5 to $100 \mathrm{kHz}$ with a field intensity of $21.8 \mathrm{kA} / \mathrm{m}$. SLP increases with increasing magnetic core size, reaching $32 \mathrm{~W} / \mathrm{g} \mathrm{Fe}_{2} \mathrm{O}_{3}$ at $100 \mathrm{kHz}$ for $16.2 \mathrm{~nm}$. Within the framework of the linear response theory, a graphical construction is proposed to describe the interplay of both size distributions and magnetic properties in the heating performance of such fluids in a given frequency range. Furthermore, a numerical model is developed to calculate the spare contribution of Néel and Brown relaxation mechanisms to $S L P$, which gives a fair reproduction of the experimental data.
\end{abstract}

DOI: 10.1103/PhysRevB.88.184406

\section{INTRODUCTION}

In the last few years a significant amount of publications have been devoted to the application of magnetic nanoparticles for hyperthermia as a tumor therapy. ${ }^{1-5}$ Once implanted, the particles can heat up the tumor by means of an external ac field, sparing surrounding healthy tissue. Compared with present methods, the use of magnetic hyperthermia is very attractive for patients as it may allow tumor removal in a less invasive way and, in addition, the nanoparticles can act as contrast agents facilitating immediate screening by magnetic resonance imaging (MRI). This simultaneous process of diagnosis and therapy corresponds to the concept of "theranostics."

Magnetic hyperthermia by direct injection of nanoparticles in prostate and glioblastoma tumors is already in clinical trials. ${ }^{1}$ An alternative route consists of the intravenous administration of magnetic nanoparticles functionalized with a biological vector. Thus tailored, the magnetic nanoparticles can recognize a therapeutic target, overexpressed in a specific tumor, and couple to it. Major challenges for this route are: (a) the heating efficiency of the nanoparticles, as a small number of them will accumulate in the tumor as compared with direct injection techniques, and (b) the particle coating, which should be biocompatible, provide aqueous solubility, and anchoring sites to attach biological vectors and/or drugs to
PACS number(s): 47.65.Cb, 75.75.Fk, 87.85.Qr, 87.19.xj the particle surface. The latter can be achieved with a polymeric coating. The use of polymers to prepare the particles and their surface leads to the formation of nanobeads, with a certain number of iron oxide nanoparticles entangled in their polymeric structure, known as "multicore" magnetic nanoparticles.

The measure of heating power of magnetic nanoparticles under an alternating magnetic field is usually referred to as specific loss power (SLP). This magnitude is expressed in energy per mass units to normalize the amount of energy loss per gram of magnetic material. The SLP depends on the structural and magnetic properties of the sample and on the magnetic field properties, being increased by increasing the intensity $H$ and the frequency $f$ of the ac external field. ${ }^{7}$ For safety reasons an upper limit for the product $f \cdot H$ (and for both $f$ and $H$ alone) has been established empirically at $4.85 \times 10^{8} \mathrm{~A} \mathrm{~m}^{-1} \mathrm{~s}^{-1}(f<1.2 \mathrm{MHz}, H<15 \mathrm{kA} / \mathrm{m}),{ }^{8}$ and so the optimal properties of the nanoparticles must be found to obtain the best performance within this permissible range.

In superparamagnetic nanoparticles, parameters to be optimized are, roughly, the anisotropy energy $E_{a}$, the saturation magnetization $M_{S}$, mean magnetic core and hydrodynamic sizes, as well as size distributions. ${ }^{7,9}$ For uniaxial nanoparticles $E_{a}$ can be written as $K_{\text {eff }}\left\langle V_{P}\right\rangle$, where $K_{\text {eff }}$ is called the effective anisotropy constant, describing both crystallographic and shape anisotropies, and $\left\langle V_{P}\right\rangle$ the mean 
volume of the magnetic cores. When no external sources are considered (such as dipolar interactions), $E_{a}$ would be the height of the energy barrier $E_{b}$ that the magnetic moment of the particle must overcome to reverse its magnetization. $M_{S}$ depends mainly on the material, although surface effects may become increasingly important as the size decreases. ${ }^{10,11}$ The size distribution of the magnetic cores will affect the $E_{a}$ distribution, while the hydrodynamic size distribution will determine the relative amount of particles that are able to rotate in a media of a certain viscosity.

Iron oxide magnetic fluids have been previously used in two different formats: as isolated particles or as multiple cores encapsulated in a nonmagnetic material. ${ }^{5,9,12}$ Authors agree that two magnetic relaxation mechanisms are involved; however, the correct use of the analytic expressions available to relate the heating efficiency with the size of the nanoparticles is still controversial. ${ }^{7,13}$ While the magnetothermic behavior of isolated nanoparticles has been the subject of interest over the past years, multicore magnetic nanoparticles have thus far not received the same attention.

The magnetic fluids analyzed here have been specifically designed for biomedical applications, with particular attention to the safety of their ingredients, showing low toxicity in cell cultures and in vivo experiments. ${ }^{14,15}$ They consist in polymeric nanobeads with a polyvinylpyridine (PVP) hydrophobic matrix entrapping a few maghemite nanoparticles randomly distributed throughout the bead volume. The nanobeads are coated with a hydrophilic polyethyleneglycol (PEG) shell that provides long-term stability to the suspension and multiple anchoring sites for their functionalization. A magnetothermal study of a series of these magnetic fluids, with variable magnetic core and hydrodynamic sizes, is reported here. This study includes structural and magnetic characterizations by means of dc magnetization and ac susceptibility as a function of temperature $(5-300 \mathrm{~K})$. In order to have a direct comparison with SLP results, ac susceptibility was measured in a wide range of frequencies $(1-70 \mathrm{kHz})$. SLP values were also obtained for a wide range of frequencies $(5-100 \mathrm{kHz})$ and a field intensity of $21.8 \mathrm{kA} / \mathrm{m}$.

Within the framework of the linear response theory (LRT), a graphical construction is proposed to describe the interplay of both size distributions and magnetic properties in the heating performance of magnetic fluids in a given frequency range. It provides a visual representation of the potential heating efficiency of a sample and the possible involvement of a Brown relaxation mechanism in the SLP, depending on the viscosity of the media. This illustrative procedure can be used in magnetic fluids made of isolated nanoparticles but is especially useful for the more complex multicore nanoparticle systems for which no analytic expressions are available. Furthermore, a numerical model based on the LRT is developed to calculate the different contributions of Néel and Brown relaxation mechanisms to the SLP. This model can be used to a priori estimate the heating performance of multicore magnetic fluids under different viscosity conditions.

\section{EXPERIMENTAL}

The magnetic fluids used in this study are the same that were studied as contrast agents for MRI in a previous work. ${ }^{16}$ As an extensive account of the methods for their preparation and characterization were given in Ref. 16, just a brief description will be given here. The preparation was carried out in several steps: ${ }^{17,18}$ (1) Synthesis of maghemite nanoparticles by in situ precipitation in a poly(4-vinylpyridine) solid matrix to obtain a maghemite/ poly(4-vinylpyridine) composite; (2) dispersion of the composite in acidic medium, then coating with PEG polymer; (3) dispersion in a phosphate-buffered saline (PBS) medium. The in situ precipitation method prevents nanoparticle agglomeration while particle size is tuned by means of the iron/polymer ratio. ${ }^{17-20}$ The coating of composite nanobeads with PEG was performed by Michael reaction between the pyridine nitrogen atoms with the acrylate at one end of the PEG chain. Two types of PEG acrylate were used: a nonfunctionalized PEG ending in -OH groups (200 Da) and a functionalized PEG with a $-\mathrm{COOH}$ terminal group (1000 Da), in a 9/1 ratio. The magnetic fluid samples were filtered through a $0.22-\mu \mathrm{m}$ membrane filter as part of the routine followed to sterilize them for in vivo experiments.

Iron content in the magnetic fluid samples was determined by atomic absorption in a plasma 40 ICP PerkinElmer spectrometer. Transmission electron microscopy (TEM) observations were carried out with a JEOL 2000-FXII microscope, cryo-TEM experiments with a FEI TECNAI F30 microscope, and dynamic light scattering (DLS) measurements in a Zetasizer Nano ZS of Malvern. The magnetic properties of these ferrofluids were studied by means of $\mathrm{dc}$ magnetization as a function of field at room temperature and ac magnetic susceptibility measurements as a function of temperature and frequency with a field amplitude of $0.3 \mathrm{kA} / \mathrm{m}$ (4 Oe) in a MPMS-XL superconducting quantum interference device (SQUID) magnetometer from Quantum Design. High-frequency susceptibility data at room temperature were obtained in a DynoMag ac susceptometer from Imego.

Hyperthermia experiments were performed on a homemade frequency-adjustable electromagnet. Technical details and measuring procedure can be found elsewhere. ${ }^{21}$ For this purpose two vessels were placed in the gap of the electromagnet, one containing $0.5 \mathrm{~mL}$ of the sample under study and another with the same volume of the dispersant (PBS) as a blank. The temperature was measured by immersing the optical probes directly in the sample and the blank vessels. The field intensity was fixed at $21.8 \mathrm{kA} / \mathrm{m}$ and the frequency dependency of the SLP measured from 5 to $100 \mathrm{kHz}$.

The heat capacity of the samples and blank was directly measured using a Q2000 differential scanning calorimeter from TA Instruments.

\section{RESULTS}

\section{A. Physical properties of the samples}

The mean sizes and standard deviations reported in Table I were obtained from the lognormal fits of the size distributions [Figs. 1(c) and 1(d)]. Figure 1(a) shows a cryo-TEM image of sample D illustrating the microstructure of the polymeric beads in stable suspension in these magnetic fluids. Larger magnification micrographs of the iron oxide nanoparticles inside the beads for this sample are shown in Fig. 1(b). The iron oxide nanoparticles have a mean size $\left\langle D_{P}\right\rangle$ that increases from $7.8 \mathrm{~nm}$ (sample A) to $16.2 \mathrm{~nm}$ (sample $\mathrm{D}$ ) in relation to the $\mathrm{Fe}_{2} \mathrm{O}_{3} / \mathrm{PVP}$ and $\mathrm{Fe}^{2+} / \mathrm{Fe}^{3+}$ ratios selected in the synthesis 
TABLE I. Characteristics of the samples.

\begin{tabular}{|c|c|c|c|c|c|c|}
\hline Sample & $\mathrm{Fe}_{2} \mathrm{O}_{3} / \mathrm{PVP}^{\mathrm{a}}$ & $\mathrm{Fe}^{2+} / \mathrm{Fe}^{3+}$ & $\left\langle D_{H}\right\rangle(\mathrm{nm})^{\mathrm{b}}$ & $\mathrm{SD}(\mathrm{nm})$ & $\left\langle D_{P}\right\rangle(\mathrm{nm})^{\mathrm{c}}$ & $\mathrm{SD}(\mathrm{nm})$ \\
\hline A & 0.5 & 0.5 & 70.1 & 11.8 & 7.8 & 0.9 \\
\hline B & 0.625 & 0.5 & 64.5 & 9.7 & 8.8 & 2.0 \\
\hline $\mathrm{C}$ & 1 & 0.5 & 104.8 & 17.4 & 10.6 & 1.6 \\
\hline $\mathrm{D}$ & 1 & 0.9 & 107.8 & 19.4 & 16.2 & 2.8 \\
\hline
\end{tabular}

${ }^{\text {a Molar ratio. }}$

${ }^{\mathrm{b}}$ Mean hydrodynamic size of beads.

${ }^{\mathrm{c}}$ Mean maghemite particle size.

of the nanoparticles (Table I). A small number of particles are elongated.

The histograms of the hydrodynamic bead size $D_{H}$ were in all cases unimodal [Fig. 1(c)]. The mean sizes of the iron oxide nanoparticles represent about $10-15 \%$ of the mean hydrodynamic sizes $\left\langle D_{H}\right\rangle$. Considering that at $\mathrm{pH}=7.4$ pyridine groups are hydrophobic and PEG residues are hydrophilic, the bead can be understood as its inside formed by folded PVP chains holding several iron oxide nanoparticles (magnetic cores) by $\mathrm{N}-\mathrm{Fe}$ coordination bonds, and the solvated PEG chains in the outside (see Fig. 2). From here on, the individual iron oxide superparamagnetic nanoparticles in the interior of the beads will be referred to as magnetic cores or simply nanoparticles; the entities dispersed in the magnetic fluid will be called multicore nanoparticles or beads.

\section{B. Magnetic characterization}

Figure 3 shows the hysteresis loops of the samples up to a field of $5 \mathrm{~T}$ at $5 \mathrm{~K}$. The inset shows the low-field region
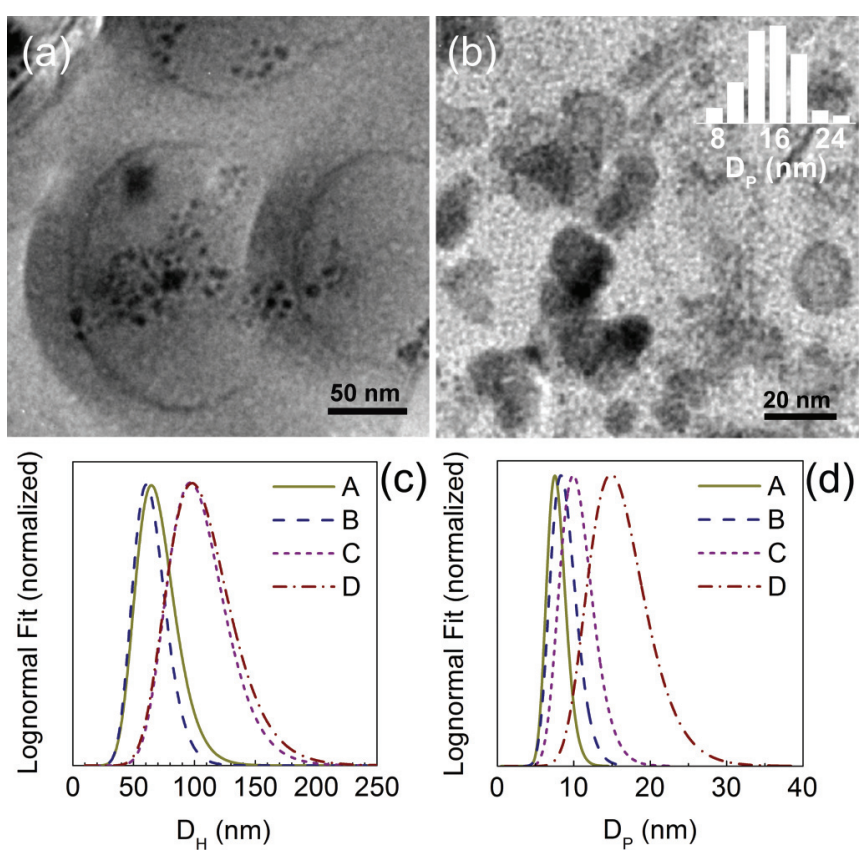

FIG. 1. (Color online) (a) Cryo-TEM and (b) TEM images of sample D showing microstructural details of these magnetic fluids. To enhance the contrast of the surface of the beads, the sample shown in (a) was conjugated with a generic antibody. (c) Hydrodynamic size distributions of beads from DLS and (d) particle size distributions from the statistical analysis of TEM images of samples A-D. of the normalized hysteresis loops $M / M_{S}$ vs $B$ (here $B=$ $\left.\mu_{0} H\right)$ with information on the remanent magnetization $M_{R}$ and coercive field $H_{C}$. Saturation magnetization values have been extracted by fitting these curves to a Langevin function, ${ }^{22}$ modified by adding a linear term to account for contributions other than ferri- or ferromagnetic in the sample (surface layer, polymer, media, etc.). ${ }^{11} M_{S}$ values are reduced compared to the one reported for maghemite nanoparticles $\left(76 \mathrm{Am}^{2} / \mathrm{kg}\right)^{10}$ and increase with $D_{P}$ (see Table II), as previously found in maghemite/PVP-like composites. ${ }^{11}$

Figure 4 shows the out-of-phase ac magnetic susceptibility $\chi^{\prime \prime}$ as a function of temperature for the set of samples. The curves have a maximum at blocking temperature $T_{B}$, which depends on the frequency of the applied field following an Arrhenius-like function (see insets in Fig. 4). At temperatures below $T_{B}$, the magnetic moment of the nanoparticles cannot reverse, i.e., it is not able to cross the energy barrier to follow the alternating field. As temperature increases, the core moments with the lowest energy barriers start to reverse by the so-called Néel relaxation. ${ }^{23}$ As expected from this model, at a given frequency, $T_{B}$ increases with the increase of $\left\langle D_{P}\right\rangle$.

In all the samples but $A, \chi^{\prime \prime}$ shows a sudden increase close to $273 \mathrm{~K}$ (see Fig. 4), which can be explained as follows. Below $273 \mathrm{~K}$ the water-based magnetic fluids are frozen and thermal activation is the only mechanism available for the moment reversal. At room temperature, the beads containing nanoparticles whose $E_{b}$ is too high for the thermal activation to be effective (say $E_{b}>k_{B} T$ ) have the possibility of reversal by

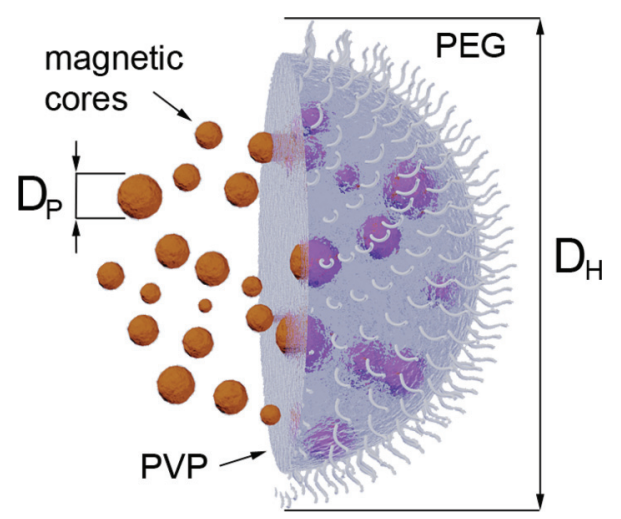

FIG. 2. (Color online) Tridimensional representation of a multicore magnetic nanoparticle. Half of the PVP matrix with the solvated PEG chains in the surface has been removed from the composition to show the iron oxide magnetic cores in the interior. Notice the difference in length scales between the nanoparticle sizes $D_{P}$ and the hydrodynamic size $D_{H}$ of the bead. 


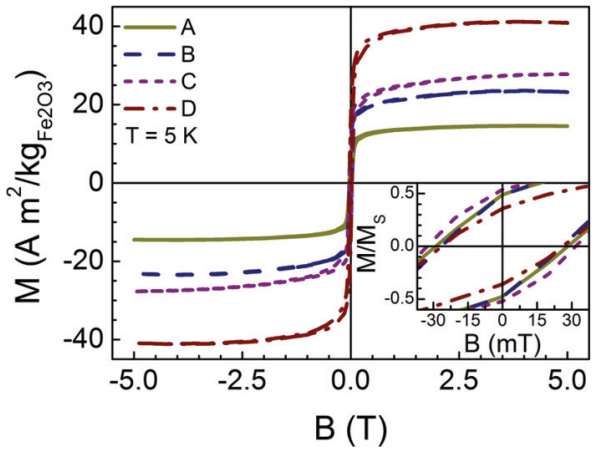

FIG. 3. (Color online) Hysteresis loops at $5 \mathrm{~K}$ for the series of samples. The inset is a zoom of the low-field region of the normalized hysteresis loop.

mechanical rotation in the fluid (termed Brownian relaxation mechanism). ${ }^{24}$ The onset of this mechanism leads to the sudden increase of $\chi^{\prime \prime}$ in samples with a relevant fraction of "large" nanoparticles (i.e., nanoparticles with $E_{b}>k_{B} T$ ).

At room temperature, the frequency dependence of $\chi^{\prime \prime}$ has a maximum around $1 \mathrm{kHz}$ in samples $\mathrm{C}$ and $\mathrm{D}$, this maximum being strongly reduced in sample B and absent in sample A (Fig. 5). To investigate the origin of this maximum and the relative importance of the Néel and Brownian mechanisms, we have prepared a new sample with $\left\langle D_{P}\right\rangle=(12.0 \pm 2.3) \mathrm{nm}$ and $\left\langle D_{H}\right\rangle=(176 \pm 53) \mathrm{nm}$ dispersed in PBS and in an agar-agar gel, the latter to constrain the bead rotation (Fig. 5 inset). Comparing $\chi^{\prime \prime}(f)$ from the fluid to that from the gel, the intensity of the signal in the second is considerably reduced and the maximum is no longer visible, leaving just a contribution that is still increasing up to $10 \mathrm{kHz}$. The "sharp" maximum in $\chi^{\prime \prime}(f)$ in the liquid samples, if related to a Néel origin, would only be compatible with an extraordinarily narrow nanoparticle size distribution (or $E_{b}$ distribution) and would not disappear in the gel sample. Thus it is safe to assume that: (1) the origin of the maximum in $\chi^{\prime \prime}(f)$ is related to the Brownian rotation of the beads; (2) there is no mechanical rotation of the particles inside the bead, since the agar-agar gel does not affect the intrabead structure; and (3) the Néel mechanism has an increasing importance at higher frequencies.

Figure 6 shows the SLP of the samples as a function of the frequency of the external field. Two commercial ferrofluids were also measured for the sake of comparison. Endorem, from Guerbet Labs, is commonly used as a contrast agent for MRI. Its structure is similar to our samples with a maghemite core diameter of $5.7 \mathrm{~nm}$ and a hydrodynamic size of the beads of $290 \mathrm{~nm} .{ }^{25}$ Chemicell's FluidMag-UC (labeled as $50 \mathrm{~nm}$ by the manufacturer) is a highly concentrated colloidal suspension of magnetite uncoated nanoparticles with mean size $8 \mathrm{~nm}$ and agglomerates with mean hydrodynamic size $32 \mathrm{~nm} .{ }^{26}$ The frequency dependence of the SLP is almost linear for all investigated ferrofluids. Energy losses increase from sample A to D, similarly to the $M_{S}$ and imaginary component of the ac susceptibility of the samples.

\section{DISCUSSION}

In magnetic fluids, the relaxation of magnetic moments has two competing mechanisms, with characteristic times $\tau_{N}$ for Néel's and $\tau_{B}$ for Brown's: ${ }^{27}$

$$
\begin{aligned}
\tau_{N} & =\tau_{0} \exp \left(\frac{K_{\text {eff }}}{k_{B} T} \frac{\pi}{6} D_{P}^{3}\right), \\
\tau_{B} & =\frac{\eta(T)}{k_{B} T} \frac{\pi}{2} D_{H}^{3},
\end{aligned}
$$

where $\tau_{0}$ is the characteristic time that describes the intrawell transitions and $\eta(T)$ is the viscosity of the media where the beads are dispersed.

The formulation in Eq. (1a) for $\tau_{N}$ was derived with the aid of the LRT and is therefore fulfilled accurately whenever the anisotropy energy is larger than the thermal energy, $\sigma=K_{\text {eff }} V_{P} /\left(k_{B} T\right) \geqslant 2$, and the measuring fields are weaker enough so that $\xi=\mu_{0} M_{S} V_{P} H /\left(k_{B} T\right) \leqslant 1 .^{13,27}$ The latter, which is the most relevant for magnetic particle hyperthermia, ensures that the field amplitude is within the linear range of the $M(H)$ response at a given frequency, i.e., far from saturation. For ac susceptibility measurements, field amplitudes of a few Oersted $(<0.5 \mathrm{kA} / \mathrm{m})$ are used as common practice and the condition on $\xi$ is satisfied for a wide range of particle volumes. In magnetothermal experiments, on the other hand, larger field amplitudes are used $(>10 \mathrm{kA} / \mathrm{m})$ and the LRT can lead to substantial underestimations of the SLP as the field amplitude (and $\xi$ ) increases. ${ }^{28}$ In a real sample, even when $\xi<1$ for particles with $V_{P} \leqslant\left\langle V_{P}\right\rangle$, there could be a significant relative amount of particles in the distribution with $V_{P}>\left\langle V_{P}\right\rangle$ where $\xi \geqslant 1$, and again, use of the LRT would lead to an underestimation of the maximum SLP attainable.

In the systems usually reported, magnetic nanoparticles are isolated and coated by a nonmagnetic shell, so that $D_{H}$ and $D_{P}$ can be univocally related by an analytic function and an

\begin{tabular}{|c|c|c|c|c|c|c|}
\hline Sample & $M_{S}^{\mathrm{a}}\left(\mathrm{Am}^{2} / \mathrm{kg}\right)$ & $M_{R} / M_{S}$ & $\mu_{0} H_{C}(\mathrm{mT})$ & $T_{B}^{\mathrm{b}}(\mathrm{K})$ & $E_{b}^{\mathrm{c}}(\mathrm{J})$ & $\tau_{0}{ }^{\mathrm{d}}(\mathrm{s})$ \\
\hline A & 13.84 & 0.48 & 28.6 & 40 & $1.0 \times 10^{-20}$ & $9.8 \times 10^{-12}$ \\
\hline B & 21.32 & 0.48 & 26.2 & 45 & $1.1 \times 10^{-20}$ & $1.5 \times 10^{-10}$ \\
\hline $\mathrm{C}$ & 25.35 & 0.53 & 32.6 & 160 & $4.7 \times 10^{-20}$ & $2.0 \times 10^{-12}$ \\
\hline $\mathrm{D}$ & 44.48 & 0.36 & 26.2 & 200 & $5.0 \times 10^{-20}$ & $5.4 \times 10^{-11}$ \\
\hline
\end{tabular}

TABLE II. Magnetic properties of the magnetic fluid samples.

${ }^{\mathrm{a}}$ From the fit of the hysteresis curve measured at $5 \mathrm{~K}$.

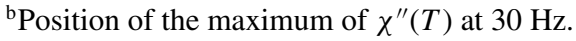

${ }^{\mathrm{c}}$ Slope and

dintercept of the linear fit of the Arrhenius plot $\ln (2 \pi f)^{-1}$ vs $\left(k_{B} T_{B}\right)^{-1}$. 

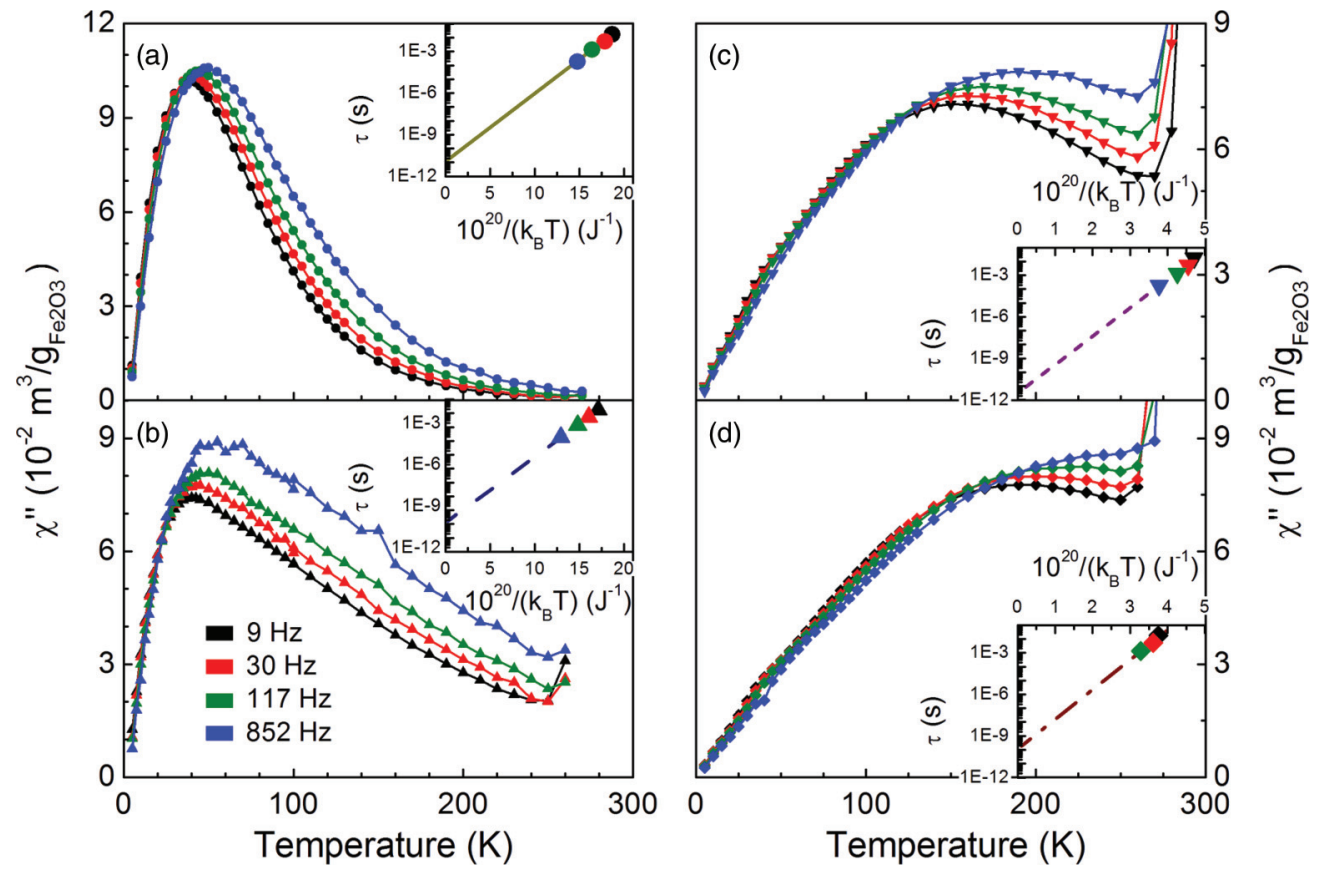

FIG. 4. (Color online) $\chi^{\prime \prime}(T)$ curves for samples A-D measured at four different frequencies. The $T_{B}$ increases with increasing $\left\langle D_{P}\right\rangle$. The insets are Arrhenius plots $\left[\tau=(2 \pi f)^{-1}\right.$ in logarithmic scale vs $\left.10^{20} /\left(k_{B} T_{B}\right)\right]$ from which linear fits $E_{b}$ and $\tau_{0}$ were extracted.

effective characteristic time can be defined as follows: ${ }^{27}$

$$
\frac{1}{\tau_{\text {eff }}}=\frac{1}{\tau_{N}}+\frac{1}{\tau_{B}}
$$

The nanostructure of the beads in the magnetic fluid samples studied here is more complex. Several magnetic cores fit inside each bead so that there are two markedly different length scales, which make difficult the writing of an analytic expression for the effective relaxation time of the entire system at a given temperature as a function of $D_{H}$ or $D_{P}$.

Having in mind that the system will always relax using the mechanism with shorter characteristic time, the most likely scenario is that, at a given temperature, in a given bead $j$ with $N_{j}$ nanoparticles inside, there will be $n_{j}$ particles with $\tau_{N_{n_{j}}}<\tau_{B_{j}}$ that relax by Néel and $\left(N_{j}-n_{j}\right)$ blocked (larger) particles that can contribute to the relaxation of the resultant bead magnetic moment by the Brownian mechanism.

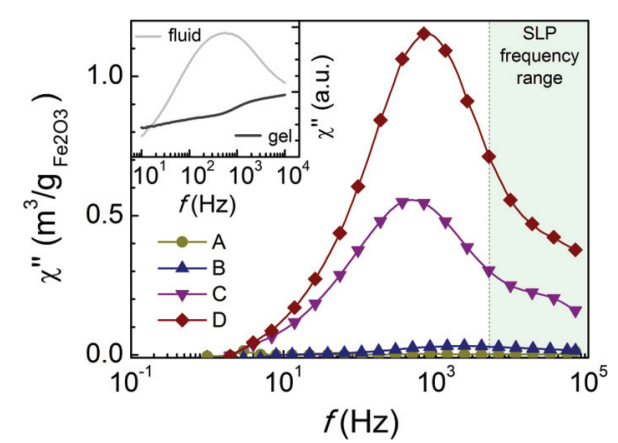

FIG. 5. (Color online) $\chi^{\prime \prime}(f)$ at $300 \mathrm{~K}$ for samples A-D. The frequency range in which the SLP studies were performed is highlighted. Inset: $\chi^{\prime \prime}(f)$ for a fluid (bright line) and gelled (dark line) sample with $\left\langle D_{P}\right\rangle=(12.0 \pm 2.3) \mathrm{nm}$ and $\left\langle D_{H}\right\rangle=(176 \pm 53) \mathrm{nm}$.

\section{A. Qualitative description of the relation between SLP and microstructure}

The increase of the SLP experimental values along these series of samples can be qualitatively explained with the help of a simple graphical construction. In this analysis, the mean magnetic core and hydrodynamic sizes as well as size distributions are the key parameters to understanding the role of the bead structure in the SLP from each sample. As for the magnetic properties the most important parameters are $E_{b}$ and $\tau_{0}$, extracted from the ac susceptibility measurements.

In each graph of Fig. 7 the normalized size distribution of nanoparticles $F_{P}\left(D_{P}\right)$ and normalized hydrodynamic size distribution of beads $F_{H}\left(D_{H}\right)$ are plotted with respect to an appropriate axis scale (lower axis for $D_{P}$, upper axis for $D_{H}$ ) to make them coincide. The Néel $\tau_{N}\left(D_{P}\right)$ and Brownian $\tau_{B}\left(D_{H}\right)$ relaxation times, calculated using Eq. (1) with the parameters in Table II and taking the viscosity of the water at $300 \mathrm{~K}$,

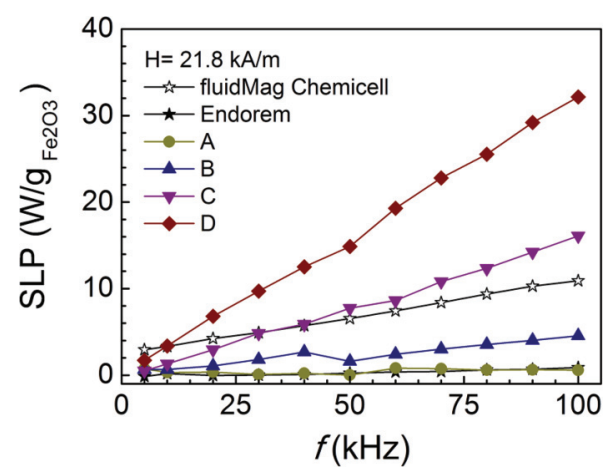

FIG. 6. (Color online) SLP as a function of frequency at field amplitude of $27.4 \mathrm{mT}$ for samples A-D and two commercial ferrofluids. 

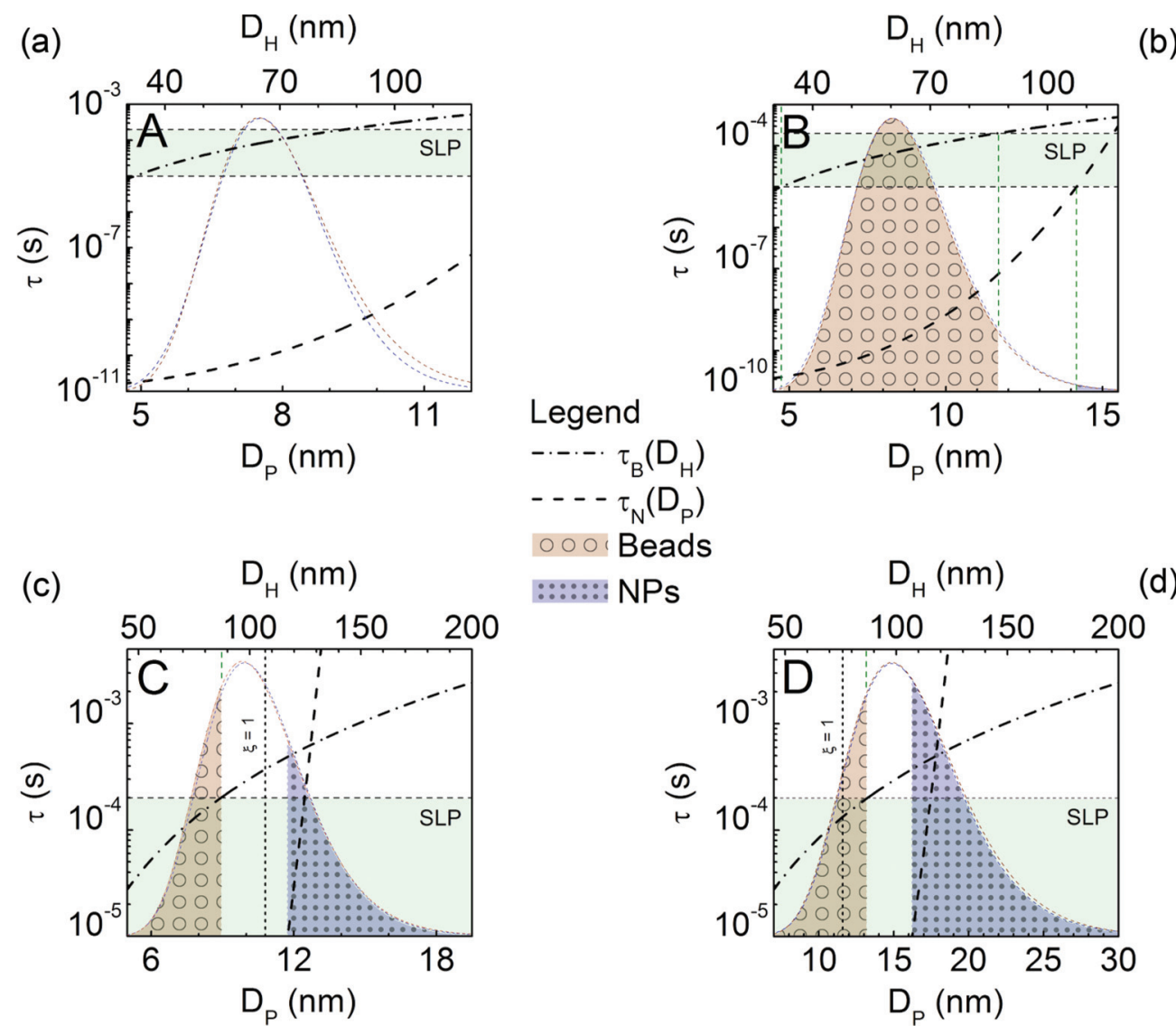

(b)

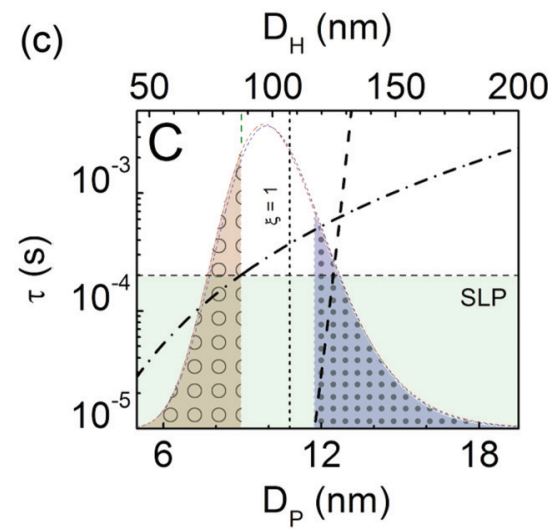

FIG. 7. (Color online) (a) Samples A, (b) B, (c) C, and (d) D. The normalized size distributions of magnetic cores $\left(D_{P}\right.$, lower axis) and hydrodynamic sizes of beads $\left(D_{H}\right.$, upper axis) for each sample are plotted with an appropriate axis scale to make them coincide with each other. Néel relaxation time of nanoparticles $\tau_{N}\left(D_{P}\right)$ (dashed line) and Brown relaxation time of beads $\tau_{B}\left(D_{H}\right)$ (dash dotted line) are plotted respectively and their values referred to the left axis. The decorated areas under the size distribution curves represent the estimated fraction of nanoparticles ( $\cdot$ pattern) and beads (o pattern) that can contribute to the SLP, according to their relaxation times, within the time window of the experiments (highlighted horizontal area). The nanoparticle size limit $\left[\xi\left(D_{P}\right) \leqslant 1\right]$ for the LRT to accurately describe $\tau_{N}\left(D_{P}\right)[\mathrm{Eq} .(1 \mathrm{a})]$ at the field amplitude of the experiments is represented (dotted line) for samples C (c) and D (d).

are also plotted for each sample. The left axis represents the time scale to which both relaxation times are referred. The time window of the SLP measurement is highlighted in each graph. The relative amounts of magnetic cores and beads in which relaxation is accessible within the time window of the experiment is represented by the dot pattern and circle pattern areas under the size distribution curves. As the reader may notice, the measurement imposes lower and upper limits to $D_{H}$ defined by the intersection of $\tau_{B}\left(D_{H}\right)$ with the boundaries of the measurement time, while for $D_{P}$ only a lower size limit is defined by $\tau_{N}\left(D_{P}\right)$ at the bottom edge of the time window. The reason for this is that all potentially Néel-blocked nanoparticles can contribute to the relaxation of their corresponding bead by Brown's mechanism.

In sample A [Fig. 7(a)] all the magnetic cores would relax by the Néel mechanism, as their characteristic time is the smallest in both length scales, but according to their size and magnetic properties, they relax too fast in order to measure any loss in that time window. Therefore, although $\tau_{B}\left(D_{H}\right)$ is within the time scale of the measurement, there are no blocked nanoparticles inside the beads to make the Brownian mechanism "effective." This is why no $\chi^{\prime \prime}(f)$ nor heating can be recorded for that sample within the time window of the SLP experiment (and, in consequence, neither circle pattern nor dot pattern areas are highlighted).

In sample B [Fig. 7(b)] the Brownian relaxation mechanism is also "accessible" for almost all the beads, but in this case those beads having magnetic cores with sizes over $14 \mathrm{~nm}$ (still blocked), at the tail of the size distribution, will account for $\chi^{\prime \prime}$ and SLP. This small contribution is weighed then by the total amount of particles (mass of magnetic material), resulting in the discrete values shown in Figs. 5 and 6, respectively.

The hydrodynamic size distributions of samples C and D are alike (Fig. 1), and the relative amounts of beads whose Brownian rotation is accessible to the magnetic relaxation of the system are also very similar [Figs. 7(c) and 7(d)]. The main differences arise when considering the relative fraction of magnetic cores that are still blocked, since sample D contains many more blocked magnetic cores. The combination of length scales $\left(D_{P}\right.$ and $\left.D_{H}\right)$ in sample D is the most favorable of the series of samples to have the larger fraction of magnetic cores contributing to energy losses in the studied frequency range, either by the Néel or the Brownian mechanisms. The fraction of magnetic cores contributing to Néel relaxation and the fraction 
of Néel-blocked magnetic cores contributing to the Brownian rotation are both larger in sample D. This sample is also the one with larger $M_{S}$ (Table II) and has shown therefore the best performance in SLP experiments.

The previous analysis of the graphics in Fig. 7 is based on the assumptions of the LRT, as we use the expression in Eq. (1a) and ignore the amplitude of the field $H$ used in the magnetothermia experiments. In order to be more precise, a vertical line at the value of $D_{P}^{*}$ at which $\xi\left(D_{P}^{*}, H\right)=1$ has been added. This line represents the size limit for the LRT to accurately describe the SLP of the samples. This means that particles with $D_{P}>D_{P}^{*}$ could be contributing to the heating of the sample and its SLP could be underestimated using the LRT. In the present samples this happens for samples $C$ and D. Nevertheless, as it can be seen in Figs. 7(c) and 7(d), this consideration has no significant impact on the results of the qualitative analysis performed in the present samples without considering it, as sample D is still the one with the most favorable size ratios.

\section{B. Estimating the Néel contribution to SLP}

Carrey et al. proposed a general method, using numerical calculations of the dynamic hysteresis loop areas (DLA), to determine the heating of magnetic nanoparticles in an alternating magnetic field. ${ }^{7}$ In the model, the crystallographic axis of the particles are assumed to be fixed, i.e., the input of the Brownian mechanism to the heating is not considered. Taking the mean sizes and magnetic properties of samples $\mathrm{C}$ and $\mathrm{D}$ (listed in Tables I and II), the SLP values can be computed using this method. As a reference and to evaluate the shortfall of the LRT approximation to compute the SLP in these samples (where $\xi>1$ for a significant amount of particles), the Néel contribution can be estimated using the formula ${ }^{7,13}$

$$
\operatorname{SLP}(f)=\pi f \mu_{0}^{2} H^{2} \int \frac{M_{S}^{2} V}{3 k_{B} T} \frac{\omega \tau_{N}}{1+\left(\omega \tau_{N}\right)^{2}} F_{P}(V) d V,
$$

where $\omega=2 \pi f$ is the frequency of the applied ac field with amplitude $H, \mu_{0}$ is the permeability of free space, and $F_{P}(V)$ is the lognormal distribution of particle volumes.

Determining the Néel sole contribution experimentally can be assessed by fixing the colloidal nanoparticles in an agar-agar gel of sufficiently high viscosity. Increasing the viscosity of the dispersing media moves the dynamic competition toward the Néel relaxation without changing any magnetic parameter in the system, and so, is representative of the actual contribution of this mechanism in normal viscosity conditions. Figure 8 shows the SLP experimental data for sample C (in fluid and in agar-agar gel) and for sample $\mathrm{D}$, together with the results of these calculations. For both samples the values obtained with the DLA reproduce quite well the linear behavior of the experimental SLP data vs frequency. As the heat produced by the samples was not measured in adiabatic conditions, the theoretical values are larger than the experimental ones as expected. The percent discrepancy in the determination of the SLP values when using the LRT approximation compared to the ones obtained with the DLA increases with the increase of the frequency up to $6 \%$ for sample $\mathrm{C}$ and $24 \%$ for sample D. Notice that D is the sample with the greater amount of nanoparticles where $\xi>1$ [Fig. 7(d)].

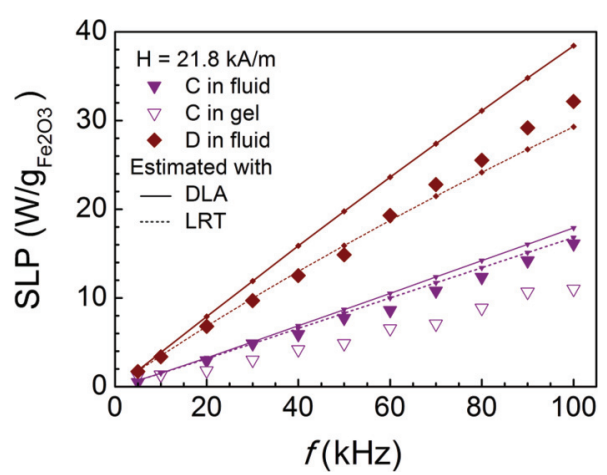

FIG. 8. (Color online) SLP experimental values (scatter) of sample $C$ in fluid $(\boldsymbol{\nabla})$ and in agar gel $(\nabla)$ and sample D in fluid $(\checkmark)$ alongside the values estimated using the DLA (solid line) and the LRT approximation (dashed line).

The drop in the heating power of sample $\mathrm{C}$ when going from fluid to gel is due to the "freezing" of the Brownian relaxation mechanism. The DLA numerical calculation used above is suitable to estimate the SLP only in samples where the particles cannot rotate. As a consequence, in order to estimate the role of the Brownian relaxation mechanism in the SLP of samples $\mathrm{C}$ and $\mathrm{D}$, a numerical simulation based on analytical equations of the LRT approximation has been developed.

\section{Estimating $\chi^{\prime \prime}$ and the SLP with a multiscale numerical model}

Within the framework of the LRT, the frequency behavior of the SLP can be expressed as proportional to the out-of-phase component of the magnetic susceptibility: 7,13

$$
\operatorname{SLP}(f)=\pi f \mu_{0} H^{2} \chi^{\prime \prime}(f) .
$$

Thus, knowing the spare contribution of each relaxation mechanism to $\chi^{\prime \prime}(f)$, the LRT can help to estimate the influence of each one on the heating of the particle assembly.

To mimic the particle system with the numerical model, over $10^{5}$ beads were created with the experimental hydrodynamic size lognormal distribution. Then, each bead was filled up to a certain volume of magnetic material with nanoparticles randomly selected from a batch created to simulate the size distribution obtained from TEM images. The volume ratio of magnetic material per hydrodynamic volume of the bead was adjusted to a value that would reasonably reproduce the average number of particles per bead observed in cryo-TEM experiments. All nanoparticles were considered spherical, their magnetic moments $\mu=M_{S} V_{P}$ and their effective anisotropy energies $E=K_{\text {eff }} V_{P}$ proportional to their volume. The saturation magnetization values were extracted from the hysteresis loops (Table II) and considered temperature independent.

To calculate the value of $\tau_{N}$ for each nanoparticle according to Eq. (1a), $\tau_{0}$ and $K_{\text {eff }}$ values were extracted from the linear fit of the Arrhenius plot, obtained with the position of the maximum of the experimental $\chi^{\prime \prime}(T)$ curves (Fig. 4) and assuming $E_{b}=K_{\text {eff }}\left\langle V_{P}\right\rangle$. For simplicity $\tau_{0}$ and $K_{\text {eff }}$ were considered constants and the possible effects of interparticle dipolar interactions ignored. These values were then fine tuned 
TABLE III. Structural and magnetic parameters used to reproduce the experimental $\chi(f)$ curves compared to those obtained from the experiments.

\begin{tabular}{lccc}
\hline \hline Parameters & & From experimental data & Used in simulations \\
\hline Sample C & $D_{P} / \mathrm{SD}$ & \multicolumn{2}{c}{$(10.6 / 1.6) \mathrm{nm}$} \\
& $D_{H} / \mathrm{SD}$ & $(104.8 / 17.4) \mathrm{nm}$ & $(106.9 / 35) \mathrm{nm}$ \\
& $E_{b}$ & $(4.7 \pm 0.5) \times 10^{-20} \mathrm{~J}$ & $5.2 \times 10^{-20} \mathrm{~J}$ \\
& $\ln \left(\tau_{0}\right)$ & $-26.9 \pm 1.5$ & -25.7 \\
Sample D & $D_{P} / \mathrm{SD}$ & $(16.2 / 2.8) \mathrm{nm}$ \\
& $D_{H} / \mathrm{SD}$ & $(107.8 / 19.4) \mathrm{nm}$ & $(96.2 / 31) \mathrm{nm}$ \\
& $E_{b}$ & $(5 \pm 1) \times 10^{-20} \mathrm{~J}$ & $5.6 \times 10^{-20} \mathrm{~J}$ \\
& $\ln \left(\tau_{0}\right)$ & $-23.6 \pm 3.5$ & -23.6 \\
\hline \hline
\end{tabular}

to obtain a better fit of the actual $\chi(f)$ experimental data measured at $300 \mathrm{~K}$ (see Table III).

To determine the magnetic susceptibility of a bead $j$ with $N_{j}$ particles, the contribution of the $n_{j}$ particles that satisfy the condition $\tau_{N_{n_{j}}}<\tau_{B_{j}}$, and therefore relax with a characteristic time $\tau_{e f f_{n_{j}}}\left(\tau_{N_{n_{j}}}, \tau_{B_{j}}\right)$ [see Eq. (2)], is added up and normalized by $n_{j}$ (the same is valid for the entire system, i.e., the contribution of every particle that relaxes by the Néel mechanism is added up and then normalized by $\sum n_{j}$ ). The contribution of the remaining "blocked" nanoparticles (if any) to the relaxation of the bead $j$ by the Brownian mechanism is calculated using the resultant magnetization and corresponding magnetic volume of such $\left(N_{j}-n_{j}\right)$ particles. The spatial orientation of the crystallographic axes is assumed randomly distributed. The Brown contribution of all beads is then added up and normalized by the number of beads that contains "blocked" nanoparticles. Finally, the total magnetic susceptibility of the system is expressed as $\chi(f)=\chi_{\text {Néel }}(f)+\chi_{\text {Brown }}(f)$.

Figure 9 shows the experimental data and simulated $\chi^{\prime \prime}(f)$ curves for samples C and D. The derived total SLP values and the contribution of each relaxation mechanism are presented in Fig. 10. The simple model used to describe the dynamics of the system reproduces quite well the frequency behavior of the magnetic susceptibility up to $5 \mathrm{kHz}$. For higher frequencies the simulated curves decrease faster than the experimental data (Fig. 9). This discrepancy can be related to the simplifications of the proposed model, which inherits the validity domain of the LRT approximations and does not take into account the effects of dipolar interparticle interactions in magnetic relaxation. Nevertheless, in the same frequency range

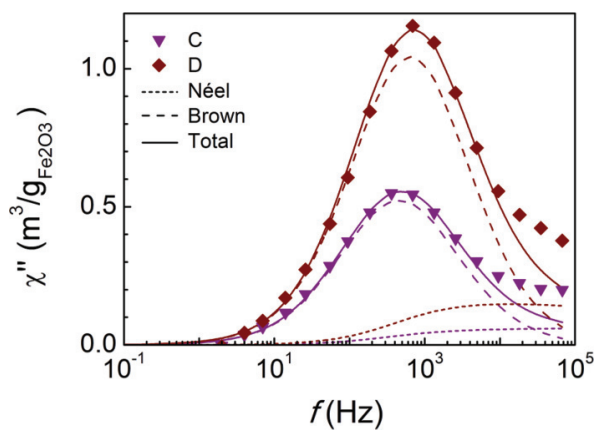

FIG. 9. (Color online) $\chi^{\prime \prime}(f)$ experimental values ( $\boldsymbol{\nabla}$ and $\left.\downarrow\right)$ and simulated curves (lines) for samples $\mathrm{C}$ and $\mathrm{D}$.

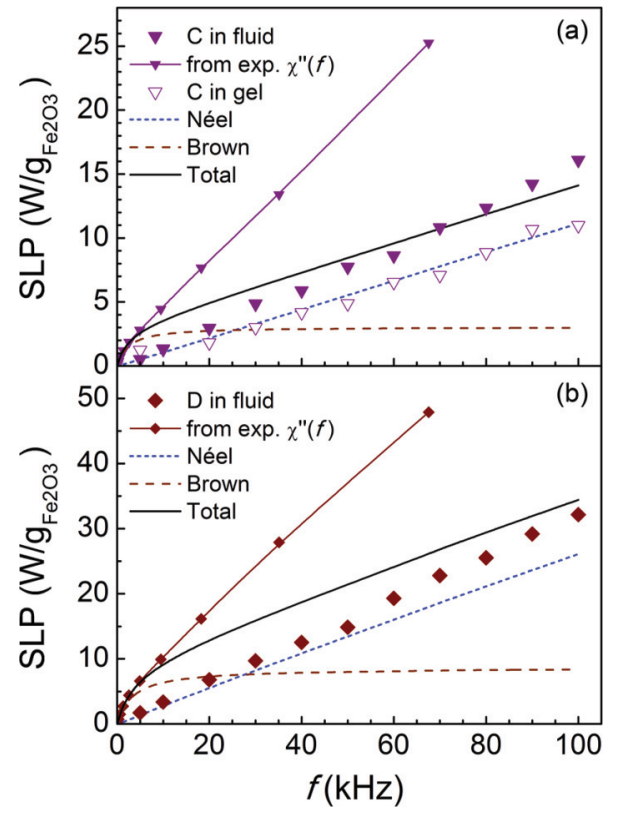

FIG. 10. (Color online) Comparative results for SLP experimental data ( $\boldsymbol{\nabla}$ and $\checkmark$ ), the values calculated using the LRT [Eq. (3)] with the experimental $\chi^{\prime \prime}(f)$ data (solid lines incorporating $\mathbf{\nabla}$ and $\bullet$ ), and those obtained from the multiscale model (lines) for (a) sample $\mathrm{C}$ and (b) sample D.

$(5-70 \mathrm{kHz})$ the heating predicted by using the LRT [Eq. (4)] with the experimental $\chi^{\prime \prime}(f)$ data is significantly larger than that recorded in hyperthermia experiments for both samples [Figs. 10(a) and 10(b)]. On the other hand, using the simulated susceptibility data reproduces quite well the experimental SLP values. Considering that the model replicates the experimental susceptibility data up to $5 \mathrm{kHz}$ (Fig. 9) and gives the best approximation of the heating performance of the samples from 5 to $70 \mathrm{kHz}$ (Fig. 10), it is likely that the experimental $\chi^{\prime \prime}(f)$ data at high frequencies overestimated the real value due to instrumental difficulties.

The contribution of the Néel relaxation mechanism to the SLP is the one that prevails at high frequencies. The input of a Brownian relaxation mechanism reaches a "saturation" value over $20 \mathrm{kHz}$ that adds to the linear growth of the Néel contribution with the increase of frequency. The experimental SLP values of sample $\mathrm{C}$ in gel are well reproduced with the Néel contribution solely [Fig. 10(a)], and by adding the Brownian relaxation mechanism input the values predicted by the model are in good agreement with the experimental SLP values of the fluid sample. Opposite to the results shown in Fig. 7, the model is able to explain the heating power of sample D using the LRT approximation by considering the contribution of both magnetic relaxation mechanisms [Fig. 10(b)].

In samples where the crystalline axis of the particles is fixed with respect to the external field, using the dynamic loop area is a more general method and gives the best estimation of the sample heating, ${ }^{7}$ while within its validity domain the LRT provides a very simple and accurate way to estimate it. In previous sections we have explained the deficiencies of the methods available so far to predict the SLP values of multicore magnetic fluids where both relaxation mechanisms interplay in two different length scales. The notable result 
represented in Fig. 10 is that, notwithstanding its simplifications, the proposed model gives the best approximation and differentiates the contributions from the Néel and Brownian relaxation mechanisms to the SLP.

\section{CONCLUSIONS}

The heating performance of a series of magnetic fluids with variable maghemite nanoparticle size and hydrodynamic size is reported. The SLP values increase throughout the series with the increase of the mean size of the magnetic cores up to $32 \mathrm{~W} / \mathrm{g}_{\mathrm{Fe}_{2} \mathrm{O}_{3}}$ at $100 \mathrm{kHz}$ with a field intensity of $21.8 \mathrm{kA} / \mathrm{m}$ for a sample with $\left\langle D_{P}\right\rangle=16.2 \mathrm{~nm}$ and $\left\langle D_{H}\right\rangle=107.8 \mathrm{~nm}$. Under the same experimental conditions, a commercial sample fluidMag-UC from Chemicell $\left(\left\langle D_{P}\right\rangle=8 \mathrm{~nm}\right)$ heated to $10 \mathrm{~W} / \mathrm{g}_{\mathrm{Fe}_{2} \mathrm{O}_{3}}$ while heating from Endorem $\left(\left\langle D_{P}\right\rangle=5.7 \mathrm{~nm}\right)$, also used as contrast agent for MRI, is negligible.

An illustrative analysis relating the nanostructural and magnetic properties of magnetic fluids within the time frame of magnetic hyperthermia measurements is presented. Considering the characteristic times of the magnetic relaxation mechanisms involved, we compare the relative amount of particles that can contribute to energy losses within the size distribution of each sample and qualitatively describe the increase of the heating performance throughout the series of samples.

Further analysis on the separate contribution of Néel and Brown relaxation mechanisms to magnetic hyperthermia in samples with larger SLP values was assessed by means of numerical simulations. Two different approaches were considered to estimate the $\operatorname{SLP}(f)$ curves: simulations of the dynamic hysteresis loop areas disregarding the Brownian relaxation mechanism (and therefore considering only the structural and magnetic parameters of the maghemite nanoparticles) and a model that replicates the $\chi(f)$ curves considering the two length scales present in the samples $\left(D_{P}\right.$ and $\left.D_{H}\right)$ and both relaxation mechanisms. While the latter has the advantage of estimating the different contribution of each relaxation mechanism to energy losses, it has the downside of having to use the LRT approximation to calculate the SLP values.

The contribution of the Néel mechanism was found to increase linearly with the increase of frequency by the two models considered and has a prominent role at higher frequencies. The contribution of the Brown mechanism reaches a plateau close to $20 \mathrm{kHz}$, adding a constant value that represents $20 \%$ of the total heating produced by samples $\mathrm{C}$ and $\mathrm{D}$ at $100 \mathrm{kHz}$.

Concerning the comparison between the multiscale model and experiments, the estimated Brownian contribution explains the different heating performance of sample $\mathrm{C}$ in gel and in fluid. The estimated joint (Néel + Brownian) contribution gives a fair description of the experimental results in sample D.

Results presented here show that these maghemite magnetic fluids, designed with special concern about their safety for biomedical purposes, have better magnetothermal efficiency than commercial ones when particle size and hydrodynamic size of the beads are tuned to 16 and $100 \mathrm{~nm}$, respectively. Samples here presented, with the above-mentioned size ratio, also showed the highest in vitro contrast efficiency for MRI, better than commercial ones. Therefore, these magnetic fluids can be considered good candidates for theranostics, i.e., its simultaneous use for diagnostic and therapeutical purposes.

\section{ACKNOWLEDGMENTS}

R.B. would like to thank ICMA-CSIC for the JAE predoc grant. Financial support from Grant No. MAT2011-25991 is gratefully acknowledged. We acknowledge Fundação para a Ciência e Tecnologia (FCT, Portugal), COMPETE, and FEDER programs (Pest-C/CTM/LA0011/2013). N.J.O.S. acknowledges FCT for the Ciência 2008 program. *rodney@unizar.es

†amillan@unizar.es

${ }^{1}$ M. Johannsen, U. Gneveckow, B. Thiesen, K. Taymoorian, C. H. Cho, N. Waldöfner, R. Scholz, A. Jordan, S. A. Loening, and P. Wust, Eur. Urol. 52, 1653 (2007); A. Jordan, R. Scholz, K. Maier-Hau, M. Johannsen, P. Wust, J. Nadobny, H. Schirra, H. Schmidt, S. Deger, S. Loening, W. Lanksch, and R. Felix, J. Magn. Magn. Mater. 225, 118 (2001).

${ }^{2}$ Q. A. Pankhurst, J. Connolly, S. K. Jones, and J. Dobson, J. Phys. D. 36, R167 (2003).

${ }^{3}$ B. Thiesen and A. Jordan, Int. J. Hyperthermia 24, 467 (2008).

${ }^{4}$ S. Laurent, S. Dutz, U. O. Häfeli, and M. Mahmoudi, Adv. Colloid Interface Sci. 166, 8 (2011).

${ }^{5}$ Challa S. S. R. Kumara and F. Mohammada, Adv. Drug Delivery Rev. 63, 789 (2011).

${ }^{6}$ V. Ozdemir, B. Williams-Jones, S. J. Glatt, M. T. Tsuang, J. B. Lohr, and C. Reist, Nat. Biotechnol. 24, 942 (2006).

${ }^{7}$ J. Carrey, B. Mehdaoui, and M. Respaud, J. Appl. Phys. 110, 039902 (2011).

${ }^{8}$ W. J. Atkinson, I. A. Brezovich, and D. P. Chakraborty, IEEE Trans. Biomed. Eng. BME-31, 70 (1984).
${ }^{9}$ R. Hergt and S. Dutz, J. Magn. Magn. Mater. 311, 187 (2007).

${ }^{10}$ J. M. D. Coey, Phys. Rev. Lett. 27, 1140 (1971).

${ }^{11}$ A. Millan, A. Urtizberea, N. J. O. Silva, F. Palacio, V. S. Amaral, E. Snoeck, and V. Serin, J. Magn. Magn. Mater. 312, L5 (2007).

${ }^{12}$ S. Dutz, M. Kettering, I. Hilger, R. Müller, and M. Zeisberger, Nanotechnology 22, 265102 (2011).

${ }^{13}$ R. Hergt, S. Dutz, and M. Zeisberger, Nanotechnology 21, 015706 (2010).

${ }^{14}$ L. M. A. Ali, M. Gutierrez, R. Cornudella, J. A. Moreno, R. Piñol, L. Gabilondo, A. Millán, and F. Palacio, J. Biomed. Nanotechnol. 9, 1272 (2013).

${ }^{15}$ R. Villa-Bellosta, G. Ibarz, A. Millán, R. Piñol, A. Ferrer-Dufol, F. Palacio, and V. Sorribas, Toxicol. Lett. 180, S221 (2008).

${ }^{16}$ H. Amiri, R. Bustamante, A. Millán, N. J. O. Silva, R. Piñol, L. Gabilondo, F. Palacio, P. Arosio, M. Corti, and A. Lascialfari, Magn. Reson. Med. 66, 1715 (2011).

${ }^{17}$ A. Millan, F. Palacio, G. Ibarz, and E. Natividad, US Patent No. US2012141602.

${ }^{18}$ R. Pinol, A. Millan, F. Palacio, and L. Gabilondo, US Patent No. WO2012045902. 
${ }^{19}$ A. Millan, F. Palacio, A. Falqui, E. Snoeck, V. Serin, A. Bhattacharjee, V. Ksenofontov, P. Gütlich, and I. Gilbert, Acta Mater. 55, 2201 (2007).

${ }^{20}$ A. Millan, A. Urtizberea, N. J. O. Silva, P. Boesecke, E. Natividad, F. Palacio, E. Snoeck, L. Soriano, A. Gutierrez, and C. Quiros, J. Appl. Crystallogr. 40, s696 (2007).

${ }^{21}$ L. M. Lacroix, J. Carrey, and M. Respaud, Rev. Sci. Instrum. 79, 093909 (2008).

${ }^{22}$ P. Langevin, Ann. Chim. Phys. 5, 70 (1905).

${ }^{23}$ L. Néel, Ann. Geophys. (CNRS) 5, 99 (1949).

${ }^{24}$ W. F. Brown, Jr., Phys. Rev. 130, 1677 (1963).
${ }^{25}$ M. F. Casula, P. Floris, C. Innocenti, A. Lascialfari, M. Marinone, M. Corti, R. A. Sperling, W. J. Parak, and C. Sangregorio, Chem. Mater. 22, 1739 (2010).

${ }^{26}$ See Supplemental Material at http://link.aps.org/supplemental/ 10.1103/PhysRevB.88.184406 for TEM and DLS results.

${ }^{27}$ M. I. Shliomis and V. I. Stepanov, in Advances in Chemical Physics: Relaxation Phenomena in Condensed Matter, edited by W. Coffey, Vol. 87 (John Wiley \& Sons, Inc., Hoboken, NJ, 1994), Chap. 1.

${ }^{28}$ Yu. L. Raikher and V. I. Stepanov, J. Magn. Magn. Mater. 320, 2692 (2008). 